\title{
Markers for Type II Collagen Breakdown Predict the Effect of Disease-Modifying Treatment on Long-Term Radiographic Progression in Patients With Rheumatoid Arthritis
}

\author{
Robert Landewé, ${ }^{1}$ Piet Geusens, ${ }^{2}$ Maarten Boers, ${ }^{3}$ Désirée van der Heijde, ${ }^{1}$ Willem Lems, ${ }^{3}$ \\ Johan te Koppele, ${ }^{4}$ Sjef van der Linden, ${ }^{1}$ and Patrick Garnero ${ }^{5}$
}

Objective. To investigate in a randomized clinical trial setting with an aggressive combination-therapy arm and a mild-monotherapy arm, whether therapyinduced changes in urinary C-terminal crosslinking telopeptide of type I collagen (CTX-I) and type II collagen (CTX-II) predict 5-year radiographic progression in patients with rheumatoid arthritis (RA).

Methods. Patients had participated in the COBRA (Combinatietherapie Bij Reumatoïde Artritis) trial comparing aggressive step-down combination therapy (the COBRA regimen, including temporary highdose prednisolone, temporary low-dose methotrexate, and sulfasalazine [SSZ]) and mild monotherapy (SSZ). Urinary CTX-I and CTX-II levels were measured at baseline and 3, 6, 9, and 12 months after initiation of treatment. Radiographs were scored according to the modified Sharp/van der Heijde method (mean of 2 independent readers who were aware of the sequence). Individual long-term radiographic progression was estimated, using baseline radiographs and all radiographs obtained during the followup period, by simple linear regression analysis (curve fitting).

Results. Both COBRA therapy and SSZ monotherapy produced a significant decrease in urinary

${ }^{1}$ Robert Landewé, MD, PhD, Désirée van der Heijde, MD, $\mathrm{PhD}$, Sjef van der Linden, MD, PhD: University Hospital Maastricht, Maastricht, The Netherlands; ${ }^{2}$ Piet Geusens, MD, PhD: University Hospital Maastricht, Maastricht, The Netherlands, and Biomedical Research Institute, Limburg University Center, Diepenbeek, Belgium; ${ }^{3}$ Maarten Boers, MD, PhD, Willem Lems, MD, PhD: Free University Medical Center, Amsterdam, The Netherlands; ${ }^{4}$ Johan te Koppele, PhD: TNO Prevention and Health, Leiden, The Netherlands; ${ }^{5}$ Patrick Garnero, PhD: INSERM Research Unit 403, and Synarc, Lyon, France.

Address correspondence and reprint requests to Robert Landewé, MD, PhD, University Hospital, Department of Internal Medicine/Rheumatology, PO Box 5800, 6202 AZ Maastricht, The Netherlands. E-mail: Rlan@sint.azm.nl.

Submitted for publication July 2, 2003; accepted in revised form January 29, 2004.
CTX-I and CTX-II levels at 3 months, and this decrease was amplified at 6 months. COBRA therapy suppressed CTX-II (change from baseline levels $-36 \%$ and $-43 \%$ at 3 and 6 months, respectively), but not CTX-I, significantly better than did SSZ $(-17 \%$ and $-21 \%$ at 3 and 6 months, respectively) at 3 and 6 months. The magnitude of the decrease in urinary CTX-II levels at 3 months significantly predicted long-term (5-year) radiographic progression $(\beta=0.48$ [95\% confidence interval $(95 \%$ CI) $0.13,0.83])$. This effect was independent of the change in disease activity and inflammation indices at 3 months. Patients whose CTX-II levels were normalized ( $<150 \mathrm{ng} / \mathrm{mmoles}$ of urinary creatinine) at 3 months had a significantly higher chance of radiographic stability (no progression over 5 years) than did patients whose CTX-II levels were increased both at baseline and at 3 months (odds ratio 4.5 [95\% CI 1.5, 13]).

Conclusion. The individual CTX-II response measured after 3 months of therapy in patients with active RA who had increased CTX-II levels at baseline independently predicts long-term radiographic progression. Urinary CTX-II levels may be used as early markers of treatment efficacy in patients with $\mathrm{RA}$.

Rheumatoid arthritis (RA) is a chronic inflammatory disease primarily affecting synovial joints, with the potential to destruct cartilage and bone, which ultimately leads to loss of function (1). The natural course of RA is heterogeneous, including patients with monophasic nondestructive oligoarthritis as well as patients with severe destructive polyarthritis. Cartilage and bone damage can be made visible on radiographs as erosions and joint space narrowing, and can be summarized and quantified in a radiographic damage score $(2,3)$. Ultimately, the level of radiographic damage appears to be associated with long-term outcome (function) (4), but radiographic progression is a slow process that evolves over many years. 
RA is treated with disease-modifying antirheumatic drugs (DMARDs) that have the potential to suppress inflammatory disease activity as well as to slow radiographic progression. The number of DMARDs registered for the treatment of RA has importantly increased over the past years. The number of therapeutic options has been boosted by the development of the tumor necrosis factor-blocking drugs etanercept, infliximab, and adalimumab. The available DMARDs have significant differences with respect to efficacy, toxicity, and price. The heterogeneous course of RA and the heterogeneous response to treatment make it pivotal for the rheumatologist to be able to predict long-term outcome as well as responsiveness to particular therapies, in order to decide on the choice of DMARD and the timing of DMARD therapy.

Predicting radiographic progression is possible at the group level, to some extent, using the following classic predictors: the presence of rheumatoid factor (RF), the level of disease activity and/or radiographic damage that is present at baseline, and the presence of the "shared epitope" (5-9). These classic predictors, however, do not suffice to adequately predict radiographic progression in individual patients.

The prediction of treatment efficacy has hardly been investigated in the past. Because clinical trials with DMARDs have shown that radiographic progression at the group level can be slowed or stopped if disease activity is suppressed, measures of disease activity, such as the erythrocyte sedimentation rate (ESR), the swollen joint count, and the Disease Activity Score (DAS) (10), are considered the best monitoring tools for guiding therapy. This approach is probably valid if DMARDs adequately suppress disease activity. However, complete disease remission in RA is rare, and monitoring disease activity does not permit differentiation in the choice and timing of administration of DMARDs.

Therefore, we need additional tools for monitoring drug responses that may predict long-term radiographic progression in individual patients. Independent of measures for disease activity, these tools should allow early differentiation between responders and nonresponders with respect to the development of future radiographic progression. It is conceivable that markers that directly and specifically reflect structural damage of cartilage and bone will be useful to monitor the effects of DMARDs with respect to future radiographic progression (11). Several biologic markers have been described in the literature, including serum hyaluronic acid, cartilage oligomeric matrix protein, and levels of the whole type I and type II collagens. However, these markers have several limitations, including lack of cartilage or bone tissue specificity and the difficulty in differentiating between the synthesis and degradation processes (11).

Type I collagen degradation can be specifically determined by measuring the level of C-terminal crosslinking telopeptide of type I collagen (CTX-I) (11), and, very recently, an immunoassay for the C-terminal crosslinking telopeptide of type II collagen (CTX-II) has become available, providing a sensitive and specific index of type II collagen degradation (12). Because type II collagen is specific for cartilage, being the most abundant constituent of cartilage matrix, and because RA is associated with type II collagen degradation, it is likely that CTX-II excretion in urine adequately reflects cartilage destruction in RA. Indeed, we recently showed in 2 independent studies that increased levels of CTX-I and CTX-II, as measured before treatment, were associated with an increased progression of radiographic damage over 1 year and 5 years, respectively, independent of disease activity and the extent of radiographic joint damage at baseline $(13,14)$. These observations suggested that patients with early RA who had increased bone and cartilage degradation, but who still had no damage visible on conventional $\mathrm{x}$-rays, will have the highest propensity to develop future radiographic damage.

The aims of the present study were 1) to investigate in a randomized clinical trial the effects of an aggressive step-down combination-therapy arm and a mild-monotherapy arm on type I and type II collagen degradation, by assessing urinary CTX-I and CTX-II levels as specific indices of bone and cartilage degradation, respectively, and 2) to investigate whether the early responses of CTX-I and CTX-II to therapy are predictive of long-term radiographic damage.

\section{PATIENTS AND METHODS}

Patients with RA. The study group comprised patients participating in the COBRA (Combinatietherapie Bij Reumatoïde Artritis) study $(15,16)$. COBRA was a 56-week multicenter clinical trial in which 155 patients who met the American College of Rheumatology (ACR; formerly, the American Rheumatism Association) criteria for RA (17) were randomly assigned to treatment. All patients had early active disease (duration $<2$ years [median 4 months]). None of the patients had been previously treated with DMARDs. One group of patients received a combination of sulfasalazine (SSZ), methotrexate, and, initially, high-dose oral prednisolone (the COBRA regimen); the other group was treated with SSZ alone. The prednisolone dosage was $60 \mathrm{mg}$ /day during the first 2 weeks and was tapered in weekly steps to the maintenance dosage of $7.5 \mathrm{mg} /$ day at week 7. Prednisolone and methotrexate were tapered and stopped after weeks 28 and 40, respec- 
Table 1. Baseline characteristics of the study patients*

\begin{tabular}{lcc}
\hline \multicolumn{1}{c}{ Characteristic } & $\begin{array}{c}\text { Sulfasalazine } \\
\text { monotherapy } \\
(\mathrm{n}=58)\end{array}$ & $\begin{array}{c}\text { COBRA } \\
\text { therapy } \\
(\mathrm{n}=52)\end{array}$ \\
\hline $\begin{array}{l}\text { Age, mean } \pm \text { SD years } \\
\text { Disease duration, mean } \pm \text { SD months }\end{array}$ & $49 \pm 12$ & $50 \pm 13$ \\
$\%$ female & $5 \pm 5$ & $5 \pm 5$ \\
$\%$ rheumatoid factor positive & 52 & 71 \\
$\%$ with erosions & 72 & 74 \\
$\%$ with the SE (absent/heterozygous/homozygous) & 44 & 42 \\
\hline
\end{tabular}

* COBRA therapy is a combination of sulfasalazine, methotrexate, and initially, high-dose oral prednisolone. $\mathrm{SE}=$ shared epitope.

tively, while SSZ was continued. After the 56-week doubleblind study, the protocol ended, and the treating physicians were free to change second-line therapy or to attempt a second tapering of methotrexate and/or prednisolone in those patients still receiving combination therapy. When possible, treatment according to the blinded protocol was continued. Urine samples (second void of the morning; nonfasting state) were obtained at baseline, 3 months, 6 months, 9 months, and at the end of the trial, and were kept frozen at $-20^{\circ} \mathrm{C}$.

The current report is based on the 110 patients for whom both urine specimens and radiographs were available at baseline and at least one followup visit (52 COBRA-treated and 58 SSZ-treated patients). These patients were followed up after the trial for a median of 4 years (range 1-6 years). During and after the trial, swollen and tender joint counts were performed, as well as an assessment of global well-being (using a visual analog scale). Disease activity was expressed as the modified DAS in 28 joints (DAS28), a validated index that includes a count of 28 joints assessed for swelling and tenderness, a measure of an acute-phase reactant (ESR), and a measure of global well-being (18).

Radiographic evaluation of the patients. Anteroposterior radiographs of the hands, wrists, and feet were obtained at baseline, week 28 , and week 56 of the randomized trial, and approximately every year thereafter. The radiographs were analyzed by 2 independent observers who were blinded to the treatment assignment but were aware of the chronologic sequence of the baseline and followup examinations. Radiographs were scored according to the modified Sharp/van der Heijde method (19). The mean value of the 2 readers' scores was used at each time point to assess joint space narrowing (JSN), bone erosion, and total Sharp score, which is the sum of the JSN and erosion scores. Simple linear regression analysis (curve fitting) (development of radiographic damage scores over time) was applied in every patient in order to get the most accurate estimate for the regression coefficient, representing the average yearly progression (expressed as Sharp units/year).

Measurement of urinary CTX-I and CTX-II. Urinary CTX-I was measured by the CrossLaps enzyme-linked immunosorbent assay (ELISA) (Nordic Bioscience, Herlev, Denmark). This assay uses a polyclonal antiserum raised against the $\beta$-isomerized EKAH $\beta$ DGGR sequence of the C-telopeptide of $\alpha 1$ chains of human type I collagen. Intraand interassay coefficients of variation were lower than $6 \%$ and $9 \%$, respectively (20).

CTX-II was measured by a new competitive ELISA (CartiLaps, Nordic Bioscience) based on a mouse monoclonal antibody raised against the EKGPDP sequence of human type II collagen C-telopeptide. This sequence, in which the lysine residue $(\mathrm{K})$ participates in crosslinking between type II collagen molecules, is present in the mature articular collagen network produced by chondrocytes. Therefore, detection of this sequence in urine is considered to specifically reflect the degradation of mature type II collagen molecules.

Intra- and interassay coefficients of variation (CVs) were lower than $8 \%$ and $10 \%$, respectively. The average intraindividual $\mathrm{CV}$ over 24 hours-reflecting diurnal variability-was only $15 \%$ (12). Urinary CTX-I and CTX-II levels were corrected by the urinary creatinine concentration, as measured by a standard colorimetric method. All measurements of CTX-I and CTX-II were performed in a central laboratory (Synarc, Lyon, France). None of the patients had marked liver and/or kidney function impairment that may have altered the urinary levels of CTX-I or CTX-II.

Statistical analysis. The CTX-I and CTX-II levels as well as the radiographic progression scores were logarithmically transformed, because both variables had a skewed distribution at all time points. Explorative analysis included time plots of CTX-I, CTX-II, the DAS28, and the ESR, stratified for treatment (SSZ or COBRA). The effects of treatment on CTX-I and CTX-II levels were expressed as the percentage change from baseline. CTX-I and CTX-II levels measured at different time points were correlated with radiographic progression, both univariately (Spearman's rank correlation) and adjusted for the ESR (partial correlation).

Linear regression analysis was performed to investigate radiographic progression as a continuous variable. Logistic regression analysis was performed to investigate radiographic progression as a binomial variable $(<2$ Sharp units/year [no progression] versus $\geq 2$ Sharp units/year [progression]).

In order to investigate the relative contribution of assessing changes in CTX-I and CTX-II levels during therapy to predicting radiographic progression, we first built a model including all known predictors of long-term progression in this cohort. These predictors included radiographic damage at baseline (present versus absent), RF status at baseline (present versus absent), disease activity at baseline (DAS28), and urinary CTX-II (or CTX-I) level at baseline. All baseline variables were assessed at the start of the trial. Thereafter, variables representing the change from baseline in CTX-I $(\Delta \mathrm{CTX}-\mathrm{I})$ or CTX-II $(\Delta \mathrm{CTX}-\mathrm{II})$ were added. In order to evaluate whether assessing changes in markers contributed more to explaining radiographic progression than to assessing changes in disease activity, variables reflecting the 3-month 

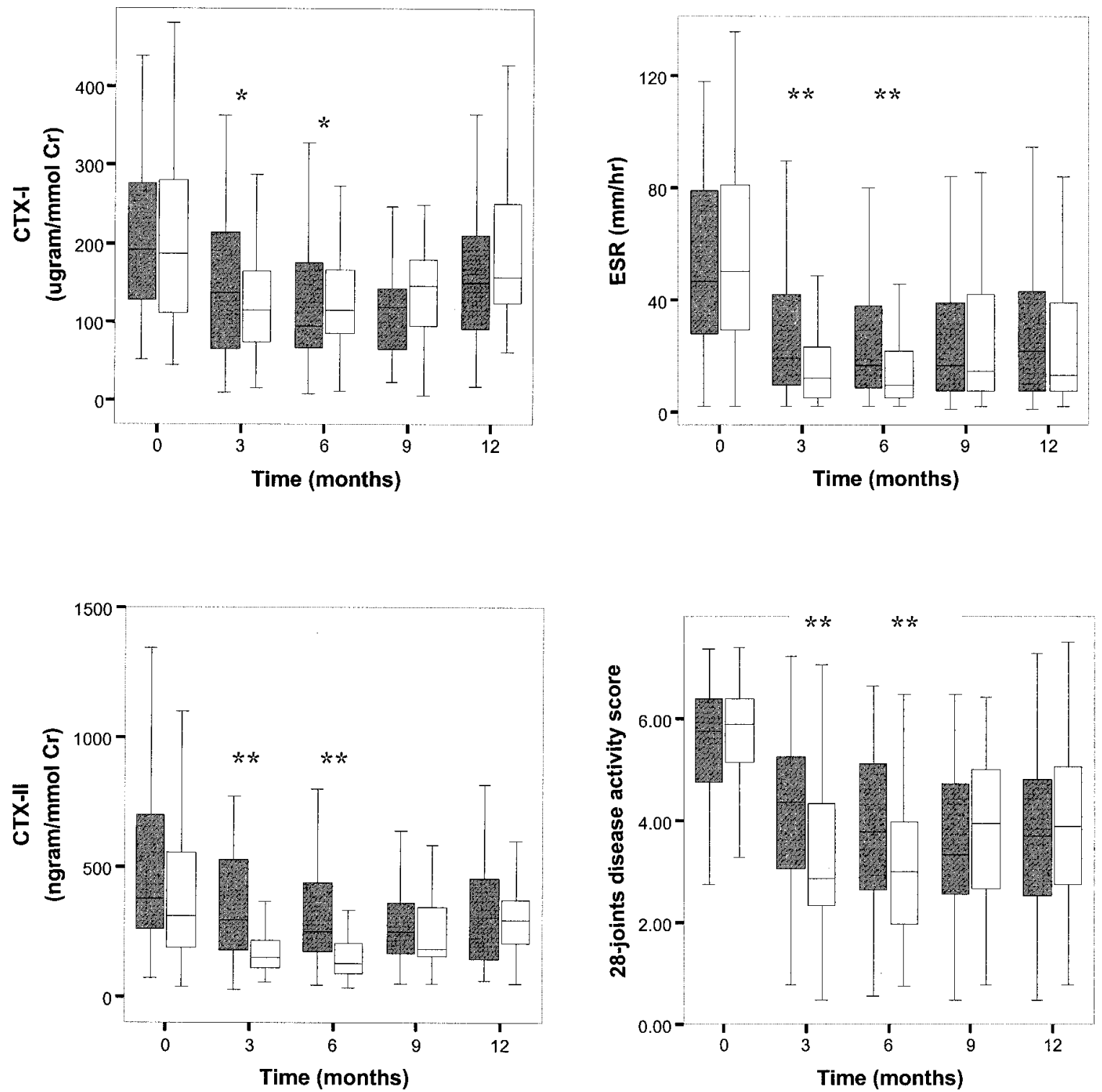

Figure 1. First-year course of biomarkers for type I and type II collagen degradation (C-terminal crosslinking telopeptide of type I [CTX-I] and type II [CTX-II] collagen), and markers for disease activity (erythrocyte sedimentation rate [ESR] and 28-joint Disease Activity Score) according to treatment at baseline (sulfasalazine [SSZ; shaded bars] or COBRA [open bars]) (see Patients and Methods for details) in patients with early rheumatoid arthritis who participated in the COBRA trial. COBRA treatment $=$ prednisolone + methotrexate + SSZ $. \mathrm{Cr}=$ creatinine. Each box represents the 25 th $/ 50$ th (median) to 75th percentiles. Lines outside the box represent the 10th and the 90th percentiles. $*=P<0.05$, within-group differences in change from baseline; $* *=P<0.01$, between-group differences in change from baseline.

change from baseline for the ESR, the swollen and tender joint counts, and the DAS28, as well as variables reflecting the presence or absence of an ACR $20 \%, 50 \%$, or $70 \%$ response (21) at 3 months, were added to the latter models one by one. In cases of linear regression analysis, residual plots were screened for linearity and homogeneity. Optimal cutoff levels for continuous predictive variables were determined by receiver operating characteristic (ROC) curve analysis. The area under the curve (AUC) was used to judge the discriminative capacity of single variables for radiographic progression (22).

\section{RESULTS}

Table 1 shows the characteristics of the study patients at baseline. All patients had active RA of very 
Table 2. Associations between urinary CTX-I and CTX-II levels measured before and during treatment and 5-year radiographic progression in patients with early $\mathrm{RA}^{*}$

\begin{tabular}{|c|c|c|c|c|}
\hline & \multicolumn{3}{|c|}{ Simple correlation } & \multirow{2}{*}{$\begin{array}{c}\text { Partial correlation } \\
\text { (controlled for ESR), } \\
\text { all patients } \\
(\mathrm{n}=104)\end{array}$} \\
\hline & $\begin{array}{l}\text { All patients } \\
(\mathrm{n}=104)\end{array}$ & $\begin{array}{l}\text { Monotherapy } \\
\quad(\mathrm{n}=56)\end{array}$ & $\begin{array}{c}\text { Combination } \\
\text { therapy } \\
(n=48)\end{array}$ & \\
\hline \multicolumn{5}{|l|}{ CTX-I } \\
\hline Baseline & 0.161 & 0.138 & 0.212 & 0.150 \\
\hline 3 months & $0.239 \dagger$ & $0.310 \dagger$ & 0.168 & 0.159 \\
\hline 6 months & 0.134 & 0.029 & $0.328+$ & 0.066 \\
\hline 9 months & 0.031 & 0.006 & 0.089 & 0.036 \\
\hline 1 year & 0.176 & 0.207 & 0.201 & 0.023 \\
\hline First year, time-averaged & - & 0.221 & $0.315 \dagger$ & 0.078 \\
\hline \multicolumn{5}{|l|}{ CTX-II } \\
\hline Baseline & $0.368 \ddagger$ & $0.267 \dagger$ & $0.447 \ddagger$ & $0.291 \S$ \\
\hline 3 months & $0.441 \ddagger$ & $0.446 \$$ & $0.442 \S$ & $0.361 \ddagger$ \\
\hline 6 months & $0.454 \ddagger$ & $0.395 \dagger$ & $0.528 \ddagger$ & $0.343 \ddagger$ \\
\hline 9 months & $0.346 \ddagger$ & $0.280 \dagger$ & $0.469 \S$ & $0.210 \dagger$ \\
\hline 1 year & $0.488 \ddagger$ & $0.452 \S$ & $0.575 \ddagger$ & $0.358 \ddagger$ \\
\hline First year, time-averaged & $0.483 \ddagger$ & $0.413 \S$ & $0.583 \ddagger$ & $0.371 \ddagger$ \\
\hline
\end{tabular}

* Values are Spearman's rank correlation coefficients. CTX-I $=$ C-terminal crosslinking telopeptide of type I collagen; CTX-II = C-terminal crosslinking telopeptide of type II collagen; RA = rheumatoid arthritis; ESR = erythrocyte sedimentation rate.

$\dagger P<0.05$

$\ddagger P<0.001$

$\S P<0.01$.

short duration. In many patients, predictors of an unfavorable prognosis were present, such as RF, erosions at baseline, and HLA-DR4 genotype. None of the patients had previously received DMARD therapy. The first explorative analysis was performed to investigate the differentiated effects of therapy on CTX-I and CTX-II levels. Figure 1 shows the levels of CTX-I and CTX-II over time, stratified for initial therapy.

The COBRA regimen and SSZ monotherapy had similar effects on the course of CTX-I levels during the first 6 months. After a decrease during the first 6 months in both groups (for SSZ, $-20 \%$ at 3 months and $-25 \%$ at 6 months; for COBRA, $-24 \%$ at 3 months and $-22 \%$ at 6 months), a rebound increase was observed after 6 months, resulting in a $17 \%$ increase in the level of CTX-I from baseline to 12 months in the COBRA group. The mean level of CTX-I in the SSZ group was still somewhat decreased $(-16 \%)$ at 12 months. The differences between the groups were not statistically significant at any time point except 12 months $(P=0.012)$.

The effects of the COBRA regimen and SSZ monotherapy on urinary CTX-II levels were different. During the first 6 months of the trial, COBRA therapy suppressed urinary CTX-II levels far better than did SSZ monotherapy (for SSZ, $-17 \%$ at 3 months and $-21 \%$ at 6 months; for COBRA, $-36 \%$ at 3 months and $-43 \%$ at 6 months). Afterwards, urinary CTX-II returned to pretreatment levels, and urinary CTX-II ex- cretion was similar in both groups at 9 and 12 months. The difference in the decrease between the SSZ and COBRA groups was statistically significant at both 3 $(P=0.002)$ and 6 months $(P<0.001)$. Figure 1 shows that CTX-II levels followed the course of disease activity parameters such as the DAS28 and the ESR during the first half year but deviated thereafter. The course of CTX-I differed in that a treatment contrast was not observed during the first 6 months.

In a second analysis, we explored whether the levels of CTX-I and CTX-II measured during therapy also correlated with radiographic progression (Table 2). CTX-I levels measured at baseline correlated only weakly with radiographic progression. CTX-I levels that were measured during treatment did not correlate very well with radiographic progression in either treatment group, and partial correlation coefficients with adjustment for the ESR were not statistically significant. The levels of CTX-II, however, correlated significantly with radiographic progression at all time points. Correlation coefficients were higher in the combination-therapy group as compared with the monotherapy group and tended to increase over time. Partial correlation coefficients were somewhat lower than bivariate correlation coefficients but were still statistically significant in all instances. These data suggest that urinary CTX-II is a determinant of long-term radiographic progression, not only at baseline but also during treatment. They also 
Table 3. Relationship between early changes in urinary CTX-II and CTX-I during treatment and long-term radiographic progression in patients with early rheumatoid arthritis*

\begin{tabular}{lccc}
\hline Change from baseline & Regression coefficient & $95 \%$ CI & $P$ \\
\hline CTX-I & & & \\
3 months & 0.01 & $-0.27,0.28$ & 0.936 \\
6 months & -0.02 & $-0.30,0.26$ & 0.873 \\
9 months & 0.03 & $-0.28,0.35$ & 0.864 \\
12 months & 0.02 & $-0.27,0.34$ & 0.768 \\
12 -month AUC & -0.03 & $-0.63,0.57$ & 0.908 \\
CTX-II & & & \\
3 months & 0.35 & $0.04,0.66$ & 0.029 \\
6 months & 0.39 & $0.09,0.69$ & 0.014 \\
9 months & 0.34 & $0.01,0.68$ & 0.044 \\
12 months & 0.23 & $-0.15,0.60$ & 0.243 \\
12-month AUC & 0.75 & $0.16,1.25$ & 0.013 \\
\hline
\end{tabular}

* C-terminal crosslinking telopeptide of type I (CTX-I) and type II (CTX-II) collagen (absolute values and area under the curve [AUC] values) and the radiographic progression rate (Radprog) were ln-transformed in all analyses. Baseline predictors of radiographic progression included in the prediction model were the erythrocyte sedimentation rate (ESR), rheumatoid factor (RF; present versus absent), radiographic damage (RD; present versus absent), and baseline CTX-I or CTX-II levels. Variables reflecting change from baseline were calculated for $3,6,9$, and 12 months, and subsequently added to the prediction model. The AUC for CTX-I and CTX-II reflects the mean CTX-I or CTX-II level over time (during the first year), which was added to the prediction model including baseline CTX-I or CTX-II. As such, the regression coefficient for the AUC (adjusted for baseline values) reflects the effects of the average change from baseline during the first year. The linear model can be expressed as follows:

$\ln$ Radprog (units/year) =

$$
\text { Constant }+\mathrm{a} \times \operatorname{ESR}(\mathrm{mm})+\mathrm{b} \times \mathrm{RF}(0,1)+\mathrm{c} \times \mathrm{RD}(0,1)+\mathrm{d} \times \ln \mathrm{CTX}_{-\mathrm{II}_{\text {baseline }}}+
$$$$
\mathrm{e} \times\left(\ln \mathrm{CTX}-\mathrm{II}_{\text {baseline }}-\ln \mathrm{CTX}-\mathrm{II}_{\mathrm{t} 3 \mathrm{~m}}\right)
$$

The term e $\times\left(\ln \mathrm{CTX}-\mathrm{II}_{\text {baseline }}-\ln \mathrm{CTX}-\mathrm{II}_{\mathrm{t} 3 \mathrm{~m}}\right)$ was replaced by e $\times\left(\ln \mathrm{CTX}-\mathrm{II}_{\mathrm{AUC}}\right)$ if $\mathrm{CTX}-\mathrm{II}_{\mathrm{AUC}}$ was used in the analyses.

suggest that this predictive capacity is independent of the effects of therapy on inflammation as assessed by the ESR.

These hypotheses were further tested in different models. The first model investigated whether changes in the levels of CTX-I or CTX-II after 3 or 6 months were predictive of 5-year radiographic progression (Table 3). The model already contained baseline variables that significantly contributed to explaining radiographic progression (ESR, RF, radiographic damage, baseline CTX-I or CTX-II levels). This model showed that adding $\triangle$ CTX-II (measured at 3, 6, or 9 months) significantly improved the prediction of long-term radiographic progression, independent of the known baseline predictors for radiographic progression (in the adjusted model, $\mathrm{R}^{2}=0.42$ for 3 months, 0.42 for 6 months, and 0.41 for 9 months). The partial $\mathrm{R}^{2}$ accounted for by $\Delta$ CTX-II was 0.05 for 3 and 6 months (if added to the model already containing the other predictors). In contrast, adding $\triangle$ CTX-I to the model did not further contribute to the prediction of radiographic progression.

We further investigated whether the improved prediction of long-term radiographic progression that was achieved by adding $\Delta$ CTX-II was independent of measures reflecting a clinical response to therapy (Table 4). The regression coefficient for the relationship between $\Delta$ CTX-II and radiographic progression $(\beta=0.48$ [ $95 \%$ confidence interval $(95 \% \mathrm{CI}) 0.13,0.83]$ ) was only slightly influenced by adding $\Delta \mathrm{ESR}, \Delta$ swollen or tender joint count, $\triangle \mathrm{DAS} 28$, or by adding the variables ACR $20 \%, 50 \%$, or $70 \%$ response. These data indicate that the predictive value of $\Delta$ CTX-II is independent of changes in measures reflecting disease activity. Correlation coefficients for the 3-month values of $\Delta$ CTX-II with changes in disease activity parameters were also calculated. $\Delta$ CTX-II was only moderately $\left(r_{s}=0.25\right)$ correlated with changes in disease activity parameters.

Using the same baseline predictors previously described, we further investigated whether we could identify patients with no long-term radiographic progression $(<2$ Sharp units/year increase versus $\geq 2$ Sharp units/year increase) by using a model including a combination of urinary CTX-II levels at baseline and 3 months. For this purpose, CTX-II was dichotomized using ROC analysis to identify the best cutoff level. ROC analysis revealed that at baseline and 3 months a CTX-II cutoff level of $150 \mathrm{ng} / \mathrm{mmole}$ of creatinine appeared to be the most adequate in discriminating 
Table 4. Confounding effects of parameters of disease activity on the predictive relationship between change from baseline in urinary CTX-II and 5-year radiographic progression*

\begin{tabular}{|c|c|c|c|c|c|c|}
\hline $\begin{array}{c}\text { Change from } \\
\text { baseline at } 3 \\
\text { months }\end{array}$ & Model 1 & Model 2 & Model 3 & Model 4 & Model 5 & Model 6 \\
\hline CTX-II & $0.48(0.13,0.83)$ & $0.43(0.05,0.81)$ & $0.55(0.19,0.91)$ & $0.44(0.08,0.80)$ & $0.42(0.06,0.78)$ & $0.48(0.10,0.86)$ \\
\hline $\begin{array}{l}\text { DAS28 } \\
\text { ESR }\end{array}$ & & $0.08(-0.08,0.24)$ & $0.01(-0.01,0.02)$ & & & \\
\hline Swollen joint count & & & & $-0.02(-0.05,0.01)$ & & \\
\hline $\begin{array}{l}\text { Tender joint count } \\
\text { HAQ score }\end{array}$ & & & & & $-0.01(-0.03,0.01)$ & $-0.02(-0.32,0.28)$ \\
\hline
\end{tabular}

* Values are the regression coefficients (95\% confidence intervals) for the variable "radiographic progression rate." Variables to test for confounding the relationship between the variables "change from baseline in urinary C-terminal crosslinking telopeptide of type II collagen (CTX-II)" and "radiographic progression rate" were added to Model 1, one after another (Models 2-6). The effect of each variable (change from baseline) on the stability of the predictive capacity of urinary CTX-II (change from baseline) was investigated. The regression coefficients for the variable "change-from-baseline" in urinary CTX-II were 0.49 after adjustment for an American College of Rheumatology (ACR) $20 \%$ response (present versus absent), 0.48 after adjustment for an ACR 50\% response, and 0.49 after adjustment for an ACR $70 \%$ response. DAS28 = Disease Activity Score in 28 joints; ESR = erythrocyte sedimentation rate; HAQ = Health Assessment Questionnaire.

patients with or without radiographic progression (ROC AUCs were 0.735 for baseline values of CTX-II and 0.767 for 3-month values of CTX-II). Using this cutoff value, $71 \%$ (25\% false negative, $4 \%$ false positive) and $74 \%$ (16\% false negative, $10 \%$ false positive) of patients were correctly classified at baseline and at 3 months, respectively. This level of $150 \mathrm{ng} / \mathrm{mmole}$ of creatinine was similar to the mean value observed in age-matched healthy controls (23).

The logistic regression model revealed that measuring CTX-II levels at 3 months adds to the prediction of radiographic progression, independent of the known baseline predictors RF status, radiographic damage, disease activity, and CTX-II (odds ratio [OR] 8.0 [95\% CI 2.5, 28.5], $P=0.001$ ).

Table 5 shows the percentage of patients with no progression after 5 years, stratified for baseline and 3-month levels of CTX-II (normal versus increased). Of primary interest for our study question is the category of patients with increased levels of CTX-II at baseline whose levels returned to normal after 3 months of therapy. Patients in this category had a significantly higher chance of having no progression after 5 years compared with patients who started with increased CTX-II levels but whose levels did not normalize during treatment.

Figure 2 shows the median levels of radiographic progression stratified for baseline and 3-month CTX-II levels. The median level of radiographic progression in patients whose CTX-II levels had normalized after 3 months of therapy was almost similar to that of patients whose levels were already normal at baseline. Figure 2 also shows that all patients with extreme levels of radiographic progression at 5 years were in the group characterized by increased CTX-II levels both at baseline and at 3 months. A logistic regression analysis of patients with an increased CTX-II level at baseline showed that patients who started with COBRA therapy had an OR of 3.8 (95\% CI 1.4, 10.5) for reaching a normalized level at 3 months, as compared with patients who started with SSZ monotherapy.

All analyses were repeated with CTX-II levels at 6 months and gave similar results. All analyses were also performed using progression of the erosion score or the joint space narrowing score, instead of the total Sharp

Table 5. Combined discriminative capacity of urinary CTX-II (absolute values), measured at baseline and 3 months after the start of therapy, for detecting patients with no long-term radiographic progression*

\begin{tabular}{lccc}
\hline $\begin{array}{c}\text { CTX-II levels } \\
\text { (baseline/3 months) }\end{array}$ & $\begin{array}{c}\text { No. of } \\
\text { patients }\end{array}$ & $\begin{array}{c}\% \text { with no } \\
\text { progression }\end{array}$ & $\begin{array}{c}\text { Odds ratio } \\
(95 \% \text { CI })\end{array}$ \\
\hline Increased/increased & 71 & 18 & 1.0 (reference) \\
Increased/normal & 20 & 50 & $4.5(1.5,13)$ \\
Normal/normal & 13 & 69 & $10(2.7,38)$ \\
\hline
\end{tabular}

* The cutoff value between normal and increased levels of C-terminal crosslinking telopeptide of type II collagen (CTX-II) is $150 \mathrm{ng} / \mathrm{mmole}$ of creatinine. The odds ratios are relative to the reference category. No progression was defined as $<2$ Sharp units/year. $95 \% \mathrm{CI}=95 \%$ confidence interval. 


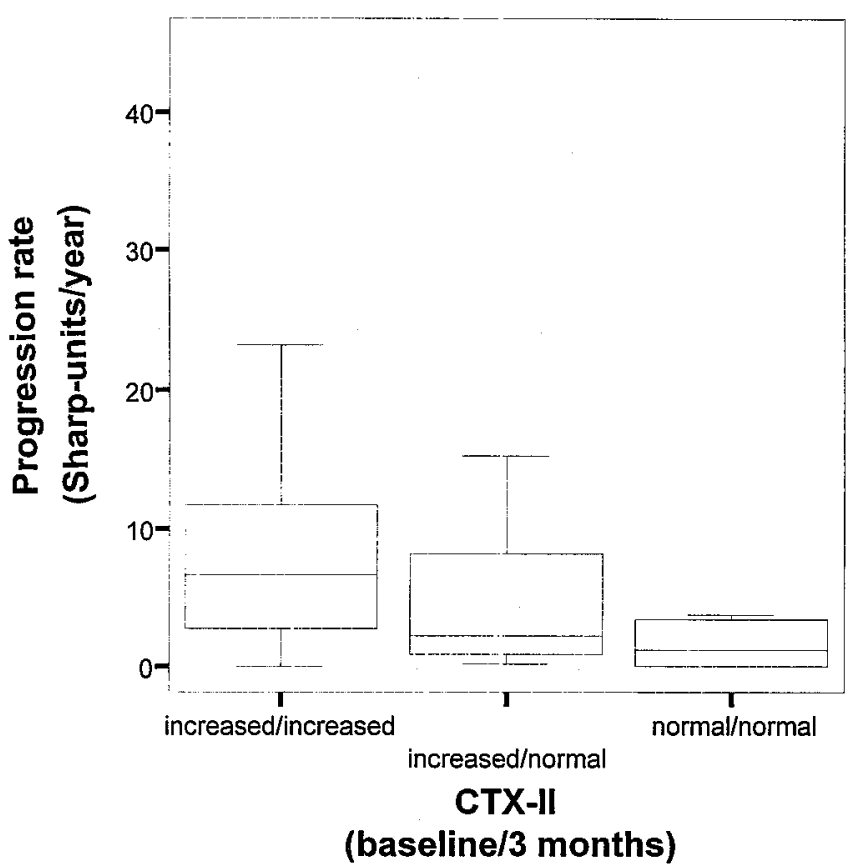

Figure 2. Radiographic progression according to the C-terminal crosslinking telopeptide of type II collagen (CTX-II) profile. CTX-II was measured at baseline and 3 months after the start of therapy. A urinary CTX-II level of $>150 \mathrm{ng} / \mathrm{mmole}$ of creatinine was considered an increased value. Each box represents the 25 th/50th (median) to 75 th percentiles. Lines outside the box represent the 10th and the 90th percentiles.

score, as dependent variables, and gave similar results (data not shown).

\section{DISCUSSION}

In this study, longitudinal patterns of markers of bone and cartilage breakdown were investigated in a randomized clinical trial using 2 different therapeutic strategies: the relatively aggressive COBRA step-down combination-therapy regimen, and relatively mild SSZ monotherapy. The COBRA regimen was better than SSZ monotherapy in suppressing urinary CTX-II levels. It was demonstrated that changes in the level of CTX-II after 3 months of therapy were predictive of long-term radiographic progression, and that this predictive effect was independent of the effects of therapy (or the type of therapy) on indices of disease activity. It was also demonstrated that the combined assessment of CTX-II at baseline and after 3 months of therapy may adequately identify patients with a favorable prognosis (i.e., no radiographic progression). All of these effects were not observed with the measurements of CTX-I.

CTX-II excretion specifically reflects type II col- lagen breakdown, which occurs specifically in cartilage. In patients with RA, cartilage degradation occurs primarily in diarthrodial peripheral joints, and CTX-II excretion may thus be considered a measure for cartilage breakdown in these joints. This makes urinary CTX-II a specific marker of joint destruction, in contrast to CTX-I, which most likely reflects overall skeletal bone turnover rather than localized joint alterations.

COBRA therapy and SSZ monotherapy were equally effective in suppressing urinary CTX-I during the first 6 months of treatment, suggesting a similar effect of the 2 treatments on bone resorption in the early phase of treatment. Afterwards, a trend toward higher CTX-I levels in the COBRA group was observed. The early phase of the COBRA regimen included a rapidly tapered high dosage of prednisolone (starting at 60 $\mathrm{mg}$ /day), which resulted in marked suppression of inflammation (15). Because inflammation is considered to be the main factor responsible for bone loss in RA $(24,25)$, the rapid suppression of inflammation by COBRA therapy is likely to explain the initial decrease of bone resorption measured by urinary CTX-I. Only at the end of the first year-3 to 6 months after the withdrawal of prednisolone-was higher excretion of CTX-I in the COBRA group observed, which may represent late adverse effects of this drug on bone metabolism.

Using several different approaches, we found a strong relationship between the dynamics of type II collagen degradation, reflected by urinary CTX-II, and long-term radiographic progression, reflected by the 5 -year progression rate. This relationship was only marginally dependent on changes in disease activity and the Health Assessment Questionnaire score (26), and, importantly, a change in disease activity itself appeared to be not significantly predictive of radiographic progression in these analyses. It was also shown that the predictive value of urinary CTX-II on long-term progression could not be accounted for by measuring a clinical response such as the ACR criteria for improvement in RA (21). These findings indicate that measurements of urinary CTX-II levels before the start of therapy and during treatment may add information with regard to long-term outcome that is not provided by serial assessments of disease activity. This observation contributes to the construct validity of CTX-II as a very early marker of forthcoming joint damage, as already suggested by results of our previous studies $(13,14)$. We have expanded these observations here by showing 1) that DMARD therapy may directly influence the level of type I and type II collagen degradation, with an impact on long-term radiographic progression, 2) that the effects 
of therapy on urinary CTX-II are partially independent of the effects of therapy on disease activity, and 3) that the accuracy of the individual prediction with regard to long-term radiographic progression can be improved by serial (at least at baseline and after 3 months) measurements of CTX-II, although it should be stressed that the proportion of misclassified patients remains considerably high.

Our study has some limitations. It was designed as a randomized controlled trial to test 2 treatment strategies, not to test the predictive value of CTX-II. Urinary CTX-I and CTX-II levels were measured retrospectively in urine samples that were frozen for this purpose, $\sim 7$ years after the start of the trial. Urine samples were available for only $70 \%$ of the patients. Prognostic similarity at baseline, therefore, cannot be assured. However, sample collection and storage, as well as measurement of CTX-I and CTX-II levels, were performed blindly with respect to treatment assignment, response to treatment, and radiographic outcome. Thus, it is highly unlikely that the patients included in this study represent biased selection with respect to longterm outcome.

The most meaningful contrast between aggressive and mild therapy was observed during the administration of corticosteroids. The observation made in this study points to an effect of corticosteroids with respect to inhibition of the breakdown of type II collagen, which is independent of the effects of corticosteroids on disease activity. Actually, a protective effect of corticosteroids on cartilage is supported by several clinical trials showing that corticosteroids are effective in reducing radiographic progression in patients with RA (27-29). However, it has to be investigated whether urinary CTX-II is also decreased by other DMARDs that are able to slow radiographic progression. An important argument against a specific effect of corticosteroids on CTX-II levels is that all of the predictive effects were also observed in the SSZ group. Finally, CTX-II excretion, which measures degradation of type II collagen (which in turn may reflect cartilage breakdown), should theoretically be associated with the progression of joint space narrowing rather than with the progression of erosions. In this study, CTX-II was associated with both, to approximately the same extent. Probably, cartilage destruction and erosions are the concurrent consequences of the same pathophysiologic processes in the joint early in the disease.

In summary, this study has shown that a patient's CTX-II profile, assessed at baseline and 3 months after the start of therapy, gives useful information with respect to the radiographic prognosis of patients with RA that cannot be obtained by measuring parameters of disease activity alone. Nonetheless, the proportion of misclassified patients is currently too high to recommend the test for clinical purposes. In the future, the CTX-II profile may be used together with other markers to judge the efficacy of a therapy or a strategy at an early stage, so that therapy can be rapidly adjusted if necessary.

\section{REFERENCES}

1. Harris ED. Rheumatoid arthritis: pathophysiology and implications for therapy. N Engl J Med 1990;322:1277-89.

2. Sharp JT. Radiologic assessment as an outcome measure in rheumatoid arthritis. Arthritis Rheum 1989;32:221-9.

3. Genant HK, Jiang Y, Peterfy C, Lu Y, Redei J, Countryman PJ. Assessment of rheumatoid arthritis using a modified scoring method on digitized and original radiographs. Arthritis Rheum 1998;41:1583-90.

4. Drossaers-Bakker KW, de Buck M, van Zeben D, Zwinderman AH, Breedveld FC, Hazes JM. Long-term course and outcome of functional capacity in rheumatoid arthritis: the effect of disease activity and radiologic damage over time. Arthritis Rheum 1999; 42:1854-60.

5. Mottonen TT. Prediction of erosiveness and rate of development of new erosion in early rheumatoid arthritis. Ann Rheum Dis 1988;47:648-53.

6. Van der Heide A, Remme CA, Hofman DM, Jacobs JW, Bijlsma JW. Prediction of progression of radiologic damage in newly diagnosed rheumatoid arthritis. Arthritis Rheum 1995;38:1466-74.

7. Jansen LM, van der Horst-Bruinsma IE, van Schaardenburg D, Bezemer PD, Dijkmans BA. Predictors of radiographic joint damage in patients with early rheumatoid arthritis. Ann Rheum Dis 2001;60:924-7

8. Combe B, Dougados M, Goupille P, Cantagrel A, Eliaou JF, Sibilia J, et al. Prognostic factors for radiographic damage in early rheumatoid arthritis: a multiparameter prospective study. Arthritis Rheum 2001;44:1736-43.

9. Van der Heijde DM, van Riel PL, van Leeuwen MA, van 't Hof MA, van Rijswijk MH, van de Putte LB. Prognostic factors for radiographic damage and physical disability in early rheumatoid arthritis: a prospective followup study of 147 patients. $\mathrm{Br} \mathrm{J}$ Rheumatol 1992;31:519-25.

10. Van der Heijde DM, van 't Hof MA, van Riel PL, Theunisse LM, Lubberts EW, van Leeuwen MA, et al. Judging disease activity in clinical practice in rheumatoid arthritis: first step in the development of a disease activity score. Ann Rheum Dis 1990;49:916-20.

11. Garnero P, Rousseau J-C, Delmas PD. Molecular basis and clinical use of biochemical markers of bone, cartilage, and synovium in joint diseases. Arthritis Rheum 2000;43:953-61.

12. Christgau S, Garnero P, Fledelius C, Moniz C, Ensig M, Gineyts E, et al. Collagen type II C-telopeptide fragments as an index of cartilage degradation. Bone 2001;29:209-15.

13. Garnero P, Gineyts E, Christgau S, Finck B, Delmas PD. Association of baseline levels of urinary glucosyl-galactosyl-pyridinoline and type II collagen C-telopeptide with progression of joint destruction in patients with early rheumatoid arthritis. Arthritis Rheum 2002;46:21-30.

14. Garnero P, Landewé R, Boers M, Verhoeven A, van der Linden S, Christgau S, et al. Association of baseline levels of markers of bone and cartilage degradation with long-term progression of joint damage in patients with early rheumatoid arthritis: the COBRA study. Arthritis Rheum 2002;46:2847-56.

15. Boers M, Verhoeven AC, Markusse HM, van de Laar MA, Westhovens R, van Denderen JC, et al. Randomised comparison 
of combined step-down prednisolone, methotrexate and sulphasalazine with sulphasalazine alone in early rheumatoid arthritis. Lancet 1997;350:301-9.

16. Landewé RB, Boers M, Verhoeven AC, Westhovens R, van de Laar MA, Markusse HM, et al. COBRA combination therapy in patients with early rheumatoid arthritis: long-term structural benefits of a brief intervention. Arthritis Rheum 2002;46:347-56.

17. Arnett FC, Edworthy SM, Bloch DA, McShane DJ, Fries JF, Cooper NS, et al. The American Rheumatism Association 1987 revised criteria for the classification of rheumatoid arthritis. Arthritis Rheum 1988;31:315-24.

18. Prevoo ML, van 't Hof MA, Kuper HH, van Leeuwen MA, van de Putte LB, van Riel PL. Modified disease activity scores that include twenty-eight-joint counts: development and validation in a prospective longitudinal study of patients with rheumatoid arthritis. Arthritis Rheum 1995;38:44-8.

19. Van der Heijde DM, van Riel PL, Nuver-Zwart IH, Gribnau FW, van de Putte LB. Effects of hydroxychloroquine and sulfasalazine on progression of joint damage in rheumatoid arthritis. Lancet 1989;1:1036-8.

20. Bonde M, Qvist P, Fledelius C, Riis BJ, Christiansen C. Immunoassay for quantifying type I collagen degradation products in urine evaluated. Clin Chem 1994;40:2022-5.

21. Felson DT, Anderson JJ, Boers M, Bombardier C, Furst D, Goldsmith C, et al. American College of Rheumatology preliminary definition of improvement in rheumatoid arthritis. Arthritis Rheum 1995;38:727-35.

22. Zweig MH, Campbell G. Receiver-operating characteristic (ROC) plots: a fundamental evaluation tool in clinical medicine. Clin Chem 1993;39:561-77.

23. Mouritzen U, Christgau S, Lehman HJ, Tanko LB, Christiansen C. Cartilage turnover assessed with a newly developed assay measuring collagen type II degradation products: influence of age, sex, menopause, hormone replacement therapy, and body mass index. Ann Rheum Dis 2003;62:332-6.

24. Gough AK, Lilley J, Eyre S, Holder RL, Emery P. Generalised bone loss in patients with early rheumatoid arthritis. Lancet 1994;344:23-7.

25. Kroot EJ, Nieuwenhuizen MG, de Waal Malefijt MC, van Riel PL, Pasker-de Jong PC, Laan RF. Change in bone mineral density in patients with rheumatoid arthritis during the first decade of the disease. Arthritis Rheum 2001;44:1254-60.

26. Fries JF, Spitz P, Kraines RG, Holman HR. Measurement of patient outcome in arthritis. Arthritis Rheum 1980;23:137-45.

27. Kirwan JR, for The Arthritis and Rheumatism Council Low-dose Glucocorticoid Study Group. The effects of glucocorticoids on joint destruction in rheumatoid arthritis. N Engl J Med 1995;333: $142-6$.

28. Mottonen T, Hannonen P, Leirisalo-Repo M, Nissila M, Kautiainen $\mathrm{H}$, Korpela $\mathrm{M}$, et al, and the FIN-RACo trial group. Comparison of combination therapy with single-drug therapy in early rheumatoid arthritis: a randomised trial. Lancet 1999;353: 1568-73.

29. Bijlsma JW, van Everdingen AA, Huisman M, de Nijs RN, Jacobs JW. Glucocorticoids in rheumatoid arthritis: effects on erosions and bone. Ann N Y Acad Sci 2002;966:82-90. 